\title{
Clinical Presentation and Factors Associated with Diabetic Ketoacidosis at the Onset of Type-1 Diabetes Mellitus in Children and Adolescent at Muhimbili National Hospital, Tanzania: A Cross Section Study
}

\author{
Honesta Kipasika ${ }^{1,2}$, Edna Majaliwa ${ }^{2}$, Benjamin Kamala ${ }^{3}$ and Lucy Mungai ${ }^{1,4}$ \\ ${ }^{1}$ Pediatric Endocrine Training Centre for Africa, Kenya \\ ${ }^{2}$ Department of Pediatrics and Child Health, Muhimbili National Hospital, Dar es Salaam, Tanzania \\ ${ }^{3}$ Department of Research, Haydom Lutheran Hospital, Tanzania \\ ${ }^{4}$ Department of Pediatrics, University of Nairobi, Kenya
}

*Corresponding author: Honesta Kipasika, Department of Pediatrics and Child Health, Muhimbili National Hospital, Dar es Salaam, P.O. Box 6500, Dar es Salaam, Tanzania, Tel: +255754592201

\begin{abstract}
Background: The incidence and clinical presentation of Type 1 diabetes mellitus (T1DM) varies markedly among countries with a worldwide trend of affected children under-5 years of age. The classical triad of polydipsia, polyuria and weight loss is the most common presentation. Similarly, the frequency of Diabetic Ketoacidosis (DKA) at the onset of T1DM is persistently high in Africa affecting more than two thirds of diabetic children.

Objective: To describe the clinical presentation of type 1 diabetes mellitus and factors associated with DKA at presentation among children aged 6 months to 19 years at Muhimbili National Hospital (MNH) in Dar es Salaam, Tanzania.

Methods: A hospital based descriptive cross-sectional study was conducted among 134 children and adolescents with T1DM at MNH diabetes clinic. Descriptive statistics such as proportions, means (SD) and median (range) were calculated. Proportions were compared by Chi-square and Fisher's exact tests. Group means for normally distributed variables were compared by Student's t-test whereas non-normal group medians were compared by non-parametric tests (Mann-Whitney-U and Kruskal-Wallis). Regression analyses were done to identify and quantify true predictors of DKA at presentation.
\end{abstract}

Results: Nearly half (46\%) of the study participants were diagnosed with DM at the age range of 5 to 10 years and almost a quarter $(23 \%)$ at the age range of $11-14$ years. Most of them presented with classical symptoms of Diabetes i.e. polyuria (95\%), polydipsia (93\%) and weight loss (79\%). Almost 4 out of 10 presented with DKA at the onset of T1DM. Age below 5 years and delayed diagnosis were the factors significantly associated with DKA at onset. Family history of DM and high education level of caretakers were found to be protective factors against DKA at diagnosis of T1DM. Mean duration of symptoms before diagnosis was 3 weeks. However, children who were diagnosed at the age between 11 to 14 years were found to have a relatively longer duration of symptoms.

Conclusion: The overall proportion of children diagnosed with DKA at the onset of T1DM was lower compared to previous study in the same hospital. Age below 5 years, delayed diagnosis and low level of education of guardian were found to be significantly associated with DKA in children with newly diagnosed T1DM.

\section{Keywords}

Type-1 DM, DKA, Clinical Presentation, Children and Adolescents

Citation: Kipasika H, Majaliwa E, Kamala B, Mungai L (2020) Clinical Presentation and Factors Associated with Diabetic Ketoacidosis at the Onset of Type-1 Diabetes Mellitus in Children and Adolescent at Muhimbili National Hospital, Tanzania: A Cross Section Study. Int J Diabetes Clin Res 7:126. doi. org/10.23937/2377-3634/1410126

Accepted: July 02, 2020: Published: July 04, 2020

Copyright: (c) 2020 Kipasika H, et al. This is an open-access article distributed under the terms of the Creative Commons Attribution License, which permits unrestricted use, distribution, and reproduction in any medium, provided the original author and source are credited. 


\section{Introduction}

Type 1 diabetes mellitus (T1DM) is a chronic disease resulting from progressive destruction of the pancreatic beta cells leading to insulin insufficiency. If left untreated, this condition progresses to metabolic derangement, with worsening hyperglycemia, ketoacidosis and death. The age at presentation is highly variable with the youngest patients diagnosed in infancy and the oldest after the fourth decade [1]. According to American diabetes association (ADA) T1DM diagnosis in children and adolescents is diagnosed whenever there are classical symptoms (polyuria, polydipsia and weight loss) of diabetes with a random blood glucose $\geq 11.1 \mathrm{mmol} / \mathrm{L}$ or fasting blood glucose $\geq 7.0 \mathrm{mmol} / \mathrm{L}$ or $\mathrm{HbA1c}>6.5 \%$ [2].

T1DM reportedly affects $15-30$ million individuals worldwide [3] with $3 \%$ of children under age of 15 years [4]. Few studies conducted in Africa have shown that the Incidence of T1DM range between $4.4 / 100,000$ in Algeria to 20/100,000 in Morocco [5]. In 1991 the incidence of type 1 diabetes mellitus in Tanzania was estimated at 1.5/100,000 [6].

There has been an increasing trend of under-five being affected worldwide with $25 \%$ of T1DM patients aged under 5 years reported in Saud Arabia [7]. Most patients have symptomatic onset of T1DM in childhood or adolescence [8] and the least after the age of 20 years [9]. The duration of symptoms prior to diagnosis can vary widely from few days to several months [1] but children and adolescent with T1DM have an abrupt clinical onset over a period of few weeks $[10,11]$.

Classical triad of polydipsia, polyuria and weight loss are the most common, but sometimes polyphagia and blurred vision may also be present [12-15]. Secondary enuresis and constipation are important non-classical symptoms at the onset of type 1 diabetes mellitus [10]. Additional symptoms may include feeling tired, growth impairment and increased susceptibility to certain infections.

Diabetes ketoacidosis (DKA) is the most serious acute complication of T1DM and frequent cause of diabetes-related death [16]. It is characterized by biochemical triad of hyperglycemia, ketonemia (ketonuria) and academia [17]. It is commonly found at onset but varies considerably among countries [18]. Studies have reported DKA prevalence at presentation to range from $21-88 \%$ of patients, with African countries topping the list $[7,15,19-23]$. Low awareness of diabetes is the biggest contributor to these high rates [5]. Multiple factors affect the risk of developing DKA at the onset of T1DM in children and adolescent. A recent study by Oren, et al. revealed that patients presenting with ketoacidosis were younger and less frequently had a first and/or second-degree relative with type 1 diabetes compared with those without diabetic ketoacidosis at presentation [24]. Furthermore, low socioeconomic status and low parental education have been found to be associated with increased risk of DKA [15].

Delayed and difficulties in diagnosing T1DM are significant causes of DKA in children with new onset disease. In a study done by Sundaram, et al. $21 \%$ of children had an average of 3 day delay in diagnosis due to missed diagnosis at the local hospital or by the general practitioner [21]. Lack of awareness among the doctors is responsible for wrong preliminary diagnosis while patient's age at the presentation also has a significant influence on diagnostic delays especially in children below 2 years [25]. Our study aimed at describing the clinical presentation of type 1 diabetes mellitus and factors associated with DKA at presentation among children aged 6 months to 19 years.

\section{Methods}

\section{Study design and participants}

This was a hospital based cross-sectional and retrospective study among children aged 6 months to 19 years at the onset of T1DM whose parents consented and children over 8 years of age gave assent to participate. The study was conducted from1st October 2018 to July 2019. Children with other chronic illnesses such as SCD and HIV were excluded.

\section{Study setting}

The study was conducted at the Muhimbili National Hospital diabetes clinic. The Hospital has bed capacity of 1500 out of which 400 beds belong to pediatric department. It has adult and pediatrics diabetes clinics. Pediatric diabetes clinic is conducted every Monday with an average of 15-30 children per day catering mostly for referred patients.

\section{Sample size and technique}

The sample size was estimated from the Kish Leslie formula (1965) using previously known proportion of DKA ( $15 \%$ by Idris, et al.) [23] At $95 \%$ level of confidence and $5 \%$ margin of error the minimum sample size estimated were 121 children. Recruitment of participants was conducted at diabetes clinic exclusively conducted on Mondays. Children meeting the inclusion criteria were enrolled consecutively until the sample size was achieved.

\section{Data collection}

After giving consent and those children over 8 years giving assent a structured questionnaire modified for this study was used for data collection. The main author administered the interview to the parents/guardians and other variables were obtained and/or confirmed from hospital records. Primary outcomes in this study included presenting symptoms diagnosis of T1DM (polydipsia, polyuria and weight loss) and DKA (presence of hyperglycemia, ketonuria, acidotic breathing and altered level of consciousness or coma). 
Other presenting symptoms included enuresis, lethargy, and polyphagia. Time taken from first symptoms to diagnosis of T1DM was recorded in weeks. Also the biodata of patients were recorded; age of patient in months, sex, and family history of DM and education level of caretaker.

\section{Data management and analysis}

Questionnaires were crosschecked after every collection. In case of any errors, correction was done immediately and before entry of data. SPSS (version 25) was used to analyze the data. Frequency tables were generated to check consistency and correction of any errors. Descriptive statistics such as proportions, means (SD) and medians (range) were calculated.

Proportions were compared by Chi-square and $\mathrm{Fi}$ sher's exact tests. Continuous variables were tested for normality by Shapiro-Wilk test. Group means for normally distributed variables were compared by Students' test whereas non-normal group median was compared by non-parametric tests (Mann-Whitney-U). Regression analyses were done to identify and quantify true predictors of outcomes. P-Value of $<0.05$ was considered significant.

\section{Ethical consideration}

Ethical clearance to conduct the study was obtained from MNH Institutional Review Board (Ref: MNH/IRB/ II/2018/012). Written informed consent was obtained from each parent/guardian of the child who met criteria before enrolling a child into the study. An assent was also sought from all children aged 8 to 19 years.
Description of the purpose of the study, it's expected outcomes, potential benefits and risks, was explained to the parents and participants. They were told that the data will be anonymous, confidential codes were used instead of names and after analysis the codes were destroyed. They were given the contacts of the principal investigator and the director for research and publication committee from Muhimbili National Hospital institutional review board.

\section{Results}

\section{Socio-demographic characteristics of respondents}

A total of 134 children were enrolled and studied from December 2018 to May 2019. The median (IQR) age at the time of this study was 14 years and six months (IQR, 10.0 and 16.7) with the mean age of 10.9 years (SD 5.4 years). Most of them were aged above 14 years with nearly equal sex distribution. Most of them had attained secondary and high level of secondary education. Other details are shown in Table 1.

At the time of diagnosis (43\%) of participants had DKA as the first presentation to the hospital. More than three quarters presented with all classical symptoms of Diabetes i.e. polyuria (95\%), polydipsia (93\%) and weight loss (79\%). Nearly half of the participants presented with Enuresis (47\%) and polyphagia (44\%). Other symptoms at the time of diagnosis are as presented in Table 2. Most of the symptoms had duration of between three weeks to four weeks by the time of diagnosis with few of them presenting within one week before being attended.

Table 1: Socio-demographic characteristics of studied children attended at MNH DM clinic from December 2018 to May 2019 (n $=134)$.

\begin{tabular}{|l|l|l|l|}
\hline Variable & Character & Count (n) & Percentage (\%) \\
\hline Age groups (years) & Less than 5 & 8 & 6 \\
\hline & 5 to 10 & 17 & 12.7 \\
\hline Sex & 11 to 14 & 37 & 27.6 \\
\hline & More than 14 & 72 & 53.7 \\
\hline Education level of the child & Male & 65 & 51.5 \\
\hline & Female & 69 & 12.7 \\
\hline & Not enrolled/Nursery & 17 & 35.8 \\
\hline Age diagnosed with DM (years) & Primary & 48 & 51.5 \\
\hline & Secondary/post sec & 69 & 22.4 \\
\hline & Mean age at DX (SD) & 30 & 45.4 \\
\hline & Less than 5 & 61 & 23.3 \\
\hline & 5 to 10 & 31 & 8.9 \\
\hline & 11 to 14 & 12 & 8.2 \\
\hline & More than 14 & 11 & 10.4 \\
\hline & Less than 1 & 14 & 35.1 \\
\hline & 1 to 2 & 47 & 46.3 \\
\hline & 3 to 5 & 62 & \\
\hline & More than 5 & & \\
\hline
\end{tabular}


Table 2: Common presenting clinical features in patients with T1DM.

\begin{tabular}{|l|l|l|l|}
\hline Symptom (s) & $\mathbf{n}(\mathbf{\%})$ & Mean duration days $\mathbf{S D}$ & $\mathbf{9 5 \%}$ Cl for the mean \\
\hline Enuresis & $62(46.3)$ & $21.7 \pm 15$ & $18.1-25.3$ \\
\hline Polyuria & $127(94.8)$ & $21.8 \pm 15$ & $19.3-24.6$ \\
\hline Polydipsia & $125(93.3)$ & $22.1 \pm 14.9$ & $19.6-24.8$ \\
\hline Weight loss & $105(78.4)$ & $24.9 \pm 14.7$ & $22.1-27.9$ \\
\hline Polyphagia & $59(44.0)$ & $26.2 \pm 17$ & $22.1-30.3$ \\
\hline Nausea & $10(7.5)$ & $7 \pm 1.7$ & $3-11.9$ \\
\hline Lethargy & $49(36.6)$ & $4.5 \pm 1.9$ & $3.1-6.06$ \\
\hline Difficulty in breathing & $59(44.0)$ & $3.1 \pm 1.7$ & $2.7-5.01$ \\
\hline Coma & $56(41.8)$ & $2.9 \pm 1.4$ & $2.3-3.9$ \\
\hline Body malaise & $41(30.5)$ & $6.2 \pm 1.4$ & $3-12.5$ \\
\hline ** Other symptoms & $40(29.8)$ & $7.8 . \pm 1.6$ & $4-11.3$ \\
\hline *Classic symptoms & $103(76.8)$ & - & - \\
\hline
\end{tabular}

*Had both, Excessive thirty, excessive urination and weight loss

*Abdominal pain, fungal infections, headache, constipation

Table 3: Bivariate and multivariate analysis of socio-demographic factors with DKA presentation among diabetic children.

\begin{tabular}{|c|c|c|c|c|c|}
\hline Variable & & Unadjusted OR CI & $P$ value & Adjusted OR Cl & $P$ value \\
\hline \multirow[t]{2}{*}{ Sex } & Male & 1 & & 1 & \\
\hline & Female & $1.47(0.74-2.56)$ & 0.271 & $1.172(0.72-4.03)$ & 0.582 \\
\hline \multirow[t]{4}{*}{ Age (years) } & $<5$ & 1 & & 1 & \\
\hline & $5-10$ & $4.0(0.68-25.0)$ & 0.125 & $4.61(0.45-47.61)$ & 0.197 \\
\hline & $11-14$ & $1.42(0.25-6.66)$ & 0.664 & $0.54(0.05-5.21)$ & 0.591 \\
\hline & $>14$ & $2.8(0.61-12.5)$ & 0.184 & $1.06(0.11-9.43)$ & 0.982 \\
\hline \multirow[t]{3}{*}{ Age at diagnosis } & $<5$ yrs & 1 & & 1 & \\
\hline & $5-10$ & $0.40(0.16-0.98)$ & 0.047 & $0.346(0.10-1.15)$ & 0.083 \\
\hline & $>10$ & $0.39(0.15-1.03)$ & 0.057 & $0.42(0.11-1.56)$ & 0.195 \\
\hline \multirow[t]{2}{*}{ Relative with DM } & No & 1 & & 1 & \\
\hline & Yes & $0.39(1.56-5.55)$ & 0.02 & $0.49(1.98-0.51)$ & 0.139 \\
\hline \multirow[t]{3}{*}{ Hospital level } & Dispensary & 1 & & 1 & \\
\hline & $\mathrm{HC}$ & $2.85(1.29-6.63)$ & 0.01 & $2.11(0.77-5.79)$ & 0.147 \\
\hline & District/Regions & $2.69(0.88-8.27)$ & 0.08 & $6.11(1.42-26.18)$ & 0.015 \\
\hline \multirow[t]{2}{*}{ Number of attendances } & Single & 1 & & 1 & \\
\hline & $>2$ & $2.52(1.24-5.15)$ & 0.011 & $2.54(1.00-6.48)$ & 0.05 \\
\hline \multirow[t]{2}{*}{ Caretaker Marital status } & Single & 1 & & 1 & \\
\hline & Married & $0.93(0.42-2.33)$ & 0.853 & $0.98(0.37-2.59)$ & 0.969 \\
\hline \multirow[t]{3}{*}{ Education of caretaker } & Primary & 1 & & 1 & \\
\hline & Secondary & $0.97(0.42-2.22)$ & 0.975 & $0.86(0.33-2.27)$ & 0.772 \\
\hline & Tertiary & $0.38(014-1.00)$ & 0.050 & $2.24(0.45-11.11)$ & 0.32 \\
\hline \multirow[t]{3}{*}{ Employment } & Unemployed & 1 & & 1 & \\
\hline & Self employed & $1.22(0.54-2.78)$ & 0.626 & $2.27(0.82-6.28)$ & 0.114 \\
\hline & Employed & $2.35(0.73-7.58)$ & 0.151 & $2.23(0.32-1.56)$ & 0.416 \\
\hline
\end{tabular}

Table 3 presents adjusted and unadjusted odds ratio for children presenting with DKA at the time of diagnosis. Sex was insignificantly associated with DKA at the time of diagnosis. Children diagnosed with T1DM at age above 5 years had nearly (60\%) lower odds of presenting with DKA as compared to those diagnosed at the age less than 5 years. Children who presented with
DKA had (60\%) lower odds of having family history of DM compared to those with no family history. Children with DKA had more than two times odds of presenting at secondary and tertiary level health facilities than dispensary level. Moreover, children who presented with DKA have more than 2.5 times increased odds of having history of visiting other health facility multiple times. 
Table 4: Distribution of socio-demographic factors with mean duration of classical diabetic symptoms among diabetic children.

\begin{tabular}{|c|c|c|c|}
\hline Character & Mean days & $95 \% \mathrm{Cl}$ of mean & *p-value \\
\hline \multicolumn{4}{|l|}{ Children characteristics } \\
\hline \multicolumn{4}{|l|}{ Age (yrs.) } \\
\hline Less than 5 & 12.6 & $5.1-19.9$ & Ref \\
\hline 5 to 10 & 24.7 & $14.1-35.4$ & 0.282 \\
\hline 11 to 14 & 21.3 & $16.8-25.9$ & 0.489 \\
\hline More than 14 & 22.4 & $18.7-26$ & 0.354 \\
\hline \multicolumn{4}{|l|}{ Sex } \\
\hline Male & 20.9 & $17.2-24.6$ & \\
\hline Female & 22.1 & $18.4-25.8$ & 0.642 \\
\hline \multicolumn{4}{|l|}{ Age diagnosed with DM } \\
\hline Less than 5 & 16.3 & $12.6-20$ & Ref \\
\hline 5 to 10 & 22.1 & $18.2-26.1$ & 0.33 \\
\hline 11 to 14 & 26.3 & 19.6-32.9 & 0.062 \\
\hline More than 14 & 22 & $12.1-31.8$ & 0.688 \\
\hline \multicolumn{4}{|l|}{ Family history of DM } \\
\hline No & 21.6 & $18.7-24.5$ & Ref \\
\hline First degree & 21.1 & $11.5-30.7$ & 0.996 \\
\hline Second degree & 22.8 & $15.9-29.7$ & 0.912 \\
\hline \multicolumn{4}{|l|}{ Level of hospital attended } \\
\hline Dispensary & 21.7 & $16.6-26.8$ & Ref \\
\hline Health centre & 22.1 & $19-25.1$ & 0.99 \\
\hline District/regional hospital & 18.5 & $4.3-32.7$ & 0.94 \\
\hline Tertiary hospital & 21.8 & $1.6-45.3$ & 0.92 \\
\hline \multicolumn{4}{|l|}{ Number of attendances } \\
\hline Single visit & 17.2 & $13.7-20.7$ & Ref \\
\hline 2 to 3 & 23.3 & $19.6-27.1$ & 0.06 \\
\hline More than 3 & 34.6 & $23.9-45.3$ & $<0.01$ \\
\hline \multicolumn{4}{|c|}{ Parent/caregiver characteristics } \\
\hline \multicolumn{4}{|l|}{ Marital status } \\
\hline Single & 16.2 & $11.6-20.7$ & Ref \\
\hline Married & 23.1 & $19.9-26.3$ & 0.273 \\
\hline Divorced & 14.5 & 8.6-37.6 & 0.997 \\
\hline
\end{tabular}

Children whose caretakers had tertiary education were (62\%) less likely to present with DKA compared with those with no formal or primary education.

Table 4 shows the duration of classical symptoms presentation in relation to social demographic characteristics. There was no effect of age and sex on the duration of presentation of classical symptoms. Children who were diagnosed at the age of between 11 to 14 years have higher chances of presenting with longer duration. Moreover, children who presented multiple times at the clinic have higher chances of presenting with longer duration compared to those with single presentation. There was no significant relationship between duration and other factors as detailed in Table 4.

\section{Discussion}

The main findings of this study shows that more than three quarters of children presented with classical DM symptoms. Nearly half presented with and enuresis and polyphagia and $43 \%$ presented with DKA at their time of diagnosis. DKA at the onset of T1DM was frequently associated with age of less than 5 years, no family history of DM, attended at tertiary centers, had visited hospital multiple times and lower level of education of the parents/caretakers. Symptoms duration was mostly between two to three weeks.

In this study, there was almost equal distribution of females (51\%) and males (49\%). Abdullah, et al. found a male preponderance, with a male:female ratio of 1.3:1 [26]. Contrary, some studies reported female preponderance $[14,15]$. This difference could be due to contextual factors and that, unlike other autoimmune disorders, T1DM do not show a strong female bias [27]. 
A large proportion of children in this study were diagnosed with DM at the age between 5 to 10 years (45.4\%) followed by the age $11-14$ years $(23 \%)$. Similar findings were observed in a study by Abdulaziz, et al. [12] whereby the age of onset peak at around 5-7 years and 11-14 years. The second peak in this study was observed to occur in the age similar to that reported by Abdullah (10-13 years) [26]. The occurrences at these age groups may support the hypothesis that the incidence of T1DM increases at puberty due to the influence of sex hormones [28].

The findings that more than three quarters presented with classical symptoms of DM at diagnosis are in line with findings in several studies across the world whereby the most common symptoms to be noticed by the family was polyuria and polydipsia $[7,10,15,19,29]$.

The finding that $43 \%$ of patients newly diagnosed with T1DM had DKA is different from previous studies in the same setting which reported $75 \%$ [30]. The reasons for this difference may be due to increased awareness of symptoms of DM in both health care professionals and general population coupled by health education and increased literacy rate. Furthermore, there is inclusion of Non-communicable diseases (NCDs) at the ministry of health strategic plans, which have contributed to improved awareness in general population at large.

Previous studies from other developing countries such as S. Africa (70\%), Congo (90\%), Benin (77\%) and Ethiopia (80\%) have reported high frequencies of DKA at T1DM diagnosis as compared to this study [14,31-33]. Differences in magnitude in different study population might be due to, the background prevalence of diabetes in the given population, presence or absence of family history of T1DM, socioeconomic status, delayed diagnosis and treatment. Also definition of DKA used and study powers to detect differences in estimate measures [31].

On the contrary, low prevalence of DKA at diagnosis of T1DM was observed in Sweden (14\%) Canada (19\%) and USA $(26 \%)[34,35]$. This may be due to high level of awareness among parents and primary physician, in addition to good and affordable health services will have early diagnosis with reduced risk of DKA in high-income countries. This is due to strong evidence for a similar relationship between life expectancy and gross domestic product (GDP) and poorer countries account for the largest share of the global burden of diseases [2].

In this study, younger age was associated with an increased risk of DKA at diagnosis. This increased risk was mostly observed in children less than 2-years-old and was still present at 5 years as compared to children above 5 yrs. Moreover, children diagnosed with T1DM at age above 5 years had lower odds of presenting with DKA as compared to those diagnosed at the age less than 5 years. This is due to increased risk for DKA in the youngest. This is in line with a Meta-analysis consisting of 32 studies reporting that children ages less than 2-years-old had 3 times the risk of presenting with DKA as children ages greater than 2-years-old. And this increased risk continued up to age 5 years [36]. Also other studies reported the same findings that younger age at T1DM diagnosis is a risk factor for DKA $[21,24,37]$. The reasons for the higher rates of DKA in the younger age groups are multifactorial. Firstly, clinicians may have a lower index of suspicion for diabetes among young children. Secondly, classical symptoms of T1DM may be subtle and not easily distinguishable from other acute illness at this age $[36,38]$. Thirdly, young children have a less developed mechanism of metabolic compensation resulting in quickly development of dehydration and acidosis $[21,22]$.

Children with DKA had higher odds of presenting more frequent at secondary and tertiary level health facilities than dispensary level. These frequent visits among children with DKA before diagnosis are perhaps due to more severe symptoms. However, this can be due to missed diagnosis at the first medical encounter for symptoms such as polyuria, polydipsia, enuresis and weight loss. Same findings were observed in Korea; a delayed diagnosis was found to increase the risk for DKA in newly diagnosed T1DM [39]. Sundaram, et al. noted that, the odds of presenting with DKA in a child with delayed diagnosis were 5.5 times higher compared to a child presenting with DKA with no delay in diagnosis [21]. Thus high index of suspicion of signs and symptoms of diabetes among healthcare providers are crucial in diagnosing T1DM hence reducing risk of DKA and its associated complications.

In this study, family history of DM was found as protective factors associated with decreased risk of DKA at disease onset. Similar findings were observed in a systemic review which showed that having a family history of T1DM was associated with decrease risk of DKA [36]. This was probably due to increased awareness and better recognition of signs and symptoms of hyperglycaemia among families with experience of diabetes. It is also possible that family history of DM alerting clinicians to an increased possibility of diabetes mellitus.

Furthermore, children whose caretakers had tertiary education were $62 \%$ less likely to present with DKA compared with those with no formal or primary education. Similarly, previous studies also shows that children from families in which parents had higher than postsecondary education or had at least one parent with academic degree are at decreased risk of a DKA at diagnosis $[36,40,41]$. Higher literacy rate is associated with health consciousness and hence likely to seek services at the health facility before the onset of DKA.

In this study, the mean duration of the symptoms prior to diagnosis was 21 days. There is no effect of age and sex on the duration of presentation of classical symptoms. Moreover, children who were diagnosed at the age of less than 5 years have higher chances of 
presenting with shorter duration. This is due to the fact that, Beta cell destruction are more aggressive in younger children because serum levels of C-Peptide are lowest in the young age at diagnosis and continue to lose their endogenous insulin secretory capacity faster than in older children [41].

This study had the following limitations. First, the definition used for DKA in this study did not include laboratory tests for blood gases; therefore, the comparison with other studies was challenging. Second, interviews involved history of past events, which subjected parents and children to recall biases. Thirdly, there were very few children under the age of 5 years, which may have led to bias the estimates.

\section{Conclusions and Recommendations}

The overall proportion of children diagnosed with DKA at the onset of T1DM was low compared to previous study. Factors associated with DKA at onset included children under-five 5 years, delayed diagnosis and parents or caretakers low level of education. Despite reduction at onset, the rate of DKA is still high hence the need for increase in public awareness on early symptoms of T1DM in order to enhance early diagnosis. Primary and secondary care providers need regular and ongoing education regarding diagnosis of T1DM and the need for immediate referral as delays in diagnosis increase risk of DKA and its associated complications.

\section{Acknowledgements}

The authors wish to thank children with T1DM and their parents/caretaker who consented to participate in the study. We acknowledge the hospital administration and diabetes clinic staff for allowing and cooperating with us to use the hospital facilities to collect data for this study. We also wish to acknowledge the study assistants for the well-done job of data collections. We thank the well-motivated data clerks for data entry and cleaning.

\section{Author's Contributions}

HK conceptualized the study design, methods, overall data curating, formal analysis and original draft preparation. BK participated in statistical design and analysis, methodology, review, editing and critically reviewed the manuscript. EM and LM conceptualized the study design, methodology and critically review of the manuscript. All authors agreed on authorship and the final submitted manuscript.

\section{Funding}

International Society of Pediatric and Adolescent Diabetes (ISPAD) funded this research. HK received an unconditional study grant from ISPAD. The funders had no role in the study design, data collection, analysis, or decision to publish or preparation of the manuscript. The corresponding author had full access to all the data in the study and had final responsibility for the decision to submit for publication.

\section{Conflict of Interests}

The authors declare no conflict of interest.

\section{References}

1. Charles GD, Clayton PE, Brown RS (2005) Brook's Clinical pediatric endocrinology. Blackwell, 596.

2. Donaghue KC, Wadwa RP, Dimeglio LA, Wong TY, Chiarelli F, et al. (2014) Microvascular and macrovascular complications in children and adolescents. Pediatr Diabetes 15: 257-269.

3. Jaakko Tuomilehto, Marjatta Karvonen, Notkola II, Elena Molchanova, Olli Taskinen (2006) Incidence and trends of childhood type 1 diabetes worldwide 1990-1999. Diabet Med 23: 857-866.

4. Ali K, Harnden A, Edge JA (2019) Type 1 diabetes in children. BMJ 342: d294.

5. Majaliwa ES, Elusiyan BEJ, Adesiyun OO, Laigong P, Adeniran AK, et al. (2008) Type 1 diabetes mellitus in the African population: Epidemiology and management challenges. Acta Biomed 79: 255-259.

6. Swai AB, Lutale JL, McLarty DG (1993) Prospective study of incidence of juvenile diabetes mellitus over 10 years in Dar es Salaam, Tanzania. BMJ 306: 1570-1572.

7. Al-Mendalawi MD, Abduljabbar MA, Aljubeh JM, Amalraj A, Cherian MP (2019) Incidence trends of childhood type 1 diabetes in Eastern Saudi Arabia. Saudi Med J 31: 10741075.

8. Harjutsalo V, Lammi N, Karvonen M, Groop P-H (2010) Age at onset of type 1 diabetes in parents and recurrence risk in offspring. Diabetes 59: 210-214.

9. Mølbak AG, Christau B, Marner B, Borch-Johnsen K, Nerup $J$ (2019) Incidence of insulin-dependent diabetes mellitus in age groups over 30 years in Denmark. Diabet Med 11: 650-655.

10. Roche EF, Menon A, Gill D, Hoey H (2005) Clinical presentation of type 1 diabetes. Pediatr Diabetes 6: 75-78.

11. Teeäär T, Liivak N, Heilman K, Kool P, Šor R, et al. (2010) Increasing incidence of childhood-onset type 1 diabetes mellitus among Estonian children in 1999-2006. Time trend analysis 1983-2006. Pediatr Diabetes 11: 107-110.

12. Al Rashed A (2011) Pattern of presentation in type 1 diabetic patients at the diabetes center of a university hospital. Ann Saudi Med 31: 243.

13. Umar $U$ (2016) Pattern of presentation of type 1 diabetic patients in Kano, Nigeria. Niger J Basic Clin Sci 13: 85.

14. Y Reddy, Ganie Y, Pillay K (2011) Characteristics of children presenting with newly diagnosed type 1 diabetes. $S$ Afr J Child Heal 7: 46-48.

15. Ibekwe M, Ibekwe R (2019) Pattern of type 1 diabetes mellitus in Abakaliki, Southeastern, Nigeria. Pediatr Call Child Heal Care 8: 59-62.

16. Wolfsdorf J, Craig M, Daneman D, Dunger D (2009) ISPAD Clinical Practice Consensus Guidelines 2009 Compendium Diabetic ketoacidosis in children and adolescents with diabetes. Pediatr Diabetes 10: 118-133.

17. Wolfsdorf J, Glaser N, Sperling MA, American Diabetes Association (2019) Diabetic ketoacidosis in infants, children, 
and adolescents: A consensus statement from the American Diabetes Association. Diabetes Care 29: 1150-1159.

18. Jennifer W (2010) In Clinic. Diabetic Ketoacidosis. Ann Intern Med 152: ITC1.

19. Ziegler R, Neu A (2018) Diabetes in childhood and adolescence. Dtsch Arztebl Int 115: 146-156.

20. Albishi LA, Altoonisi MM, Alblewi SM, Osman RH, Ahmed NA, et al. (2017) Clinical demographic patterns of type 1 diabetes in Saudi children in Tabuk City, 2000-2010. Journal of Diabetes Mellitus 7: 41-54.

21. Sundaram PCB, Day E, Kirk JMW (2009) Delayed diagnosis in type 1 diabetes mellitus. Arch Dis Child 94: 151-152.

22. Atkilt HS, Turago MG, Tegegne BS (2017) Clinical characteristics of diabetic ketoacidosis in children with newly diagnosed type 1 diabetes in Addis Ababa, Ethiopia: A cross-sectional study. PLoS One 44: 7-9.

23. Idris $U$ (2018) Childhood diabetes mellitus in a rural tertiary hospital in North-West Nigeria. CHRISMED J Heal Res 5: 123-126.

24. de Vries L, Oren L, Lazar L, Lebenthal Y, Shalitin S, et al (2013) Factors associated with diabetic ketoacidosis at onset of type 1 diabetes in children and adolescents. Diabet Med 30: 1360-1366.

25. Pawlowicz M, Birkholz D, Niedzwiecki M, Balcerska A (2009) Difficulties or mistakes in diagnosing type 1 diabetes in children? Demographic factors influencing delayed diagnosis. Pediatr Diabetes 10: 542-549.

26. Abdullah MA (2019) Epidemiology of type I diabetes mellitus among Arab children. Saudi Med J 26: 911-917.

27. Gale EAM, Gillespie KM (2001) Diabetes and gender. Diabetologia 44: 3-15.

28. Chowdhury S (2015) Puberty and type 1 diabetes. Indian J Endocrinol Metab 19: S51-S54.

29. Lévy-Marchal C, Patterson CC, Green A, EURODIAB ACE Study Group. Europe and Diabetes (2001) Geographical variation of presentation at diagnosis of type I diabetes in children: The EURODIAB study. European and Dibetes. Diabetologia 44: B75-B80.

30. Majaliwa ES, Munubhi E, Ramaiya K, Mpembeni $R$, Sanyiwa A, et al. (2007) Survey on acute and chronic complications in children and adolescents with type 1 diabetes at Muhimbili National Hospital in Dar es Salaam, Tanzania. Diabetes Care 30: 2187-2192.
31. Otieno CF, Kayima JK, Omonge EO, Oyoo GO (2005) Diabetic ketoacidosis: Risk factors, mechanisms and management strategies in sub-Saharan Africa: A review. East Afr Med J 82: S197-S203.

32. Onyiriuka AN, Ifebi E (2013) Ketoacidosis at diagnosis of type 1 diabetes in children and adolescents: Frequency and clinical characteristics. J Diabetes Metab Disord 12: 47.

33. Fantahun B, Gedlu E (2008) Prevalence of diabetic ketoacidosis in newly diagnosed diabetes mellitus pediatric patients in Tikur Anbessa specialized hospital. Ethiop J Pediatr Child Heal 4: 1-8.

34. Hanas R, Lindgren F, Lindblad B (2007) Diabetic ketoacidosis and cerebral oedema in Sweden--a 2-year paediatric population study. Diabet Med 24: 1080-1085.

35. Bui H, To T, Stein R, Fung K, Daneman D (2010) Is diabetic ketoacidosis at disease onset a result of missed diagnosis? J Pediatr 156: 472-477.

36. Usher-smith JA, Thompson MJ, Sharp SJ (2018) Factors associated with the presence of diabetic ketoacidosis at diagnosis of diabetes in children and young adults : A systematic review.

37. Gruber N, Reichman B, Lerner-Geva L, Pinhas-Hamiel O (2015) Increased risk of severe diabetic ketoacidosis among Jewish ultra-orthodox children. Acta Diabetol 52: 365-371.

38. Jefferies C, Cutfield SW, Derraik JGB, Bhagvandas J, Albert BB, et al. (2015) 15-year incidence of diabetic ketoacidosis at onset of type 1 diabetes in children from a regional setting (Auckland, New Zealand). Sci Rep 5: 10358.

39. Lee HJ, Yu HW, Jung HW, Lee YA, Kim JH, et al. (2017) Factors associated with the presence and severity of diabetic ketoacidosis at diagnosis of type 1 diabetes in Korean children and adolescents. J Korean Med Sci 32: 303-309.

40. Rewers A, Klingensmith G, Davis C, Petitti DB, Pihoker C, et al. (2008) Presence of diabetic ketoacidosis at diagnosis of diabetes mellitus in youth: The search for diabetes in youth study. Pediatrics 121: e1258-e1266.

41. Komulainen J, Kulmala P, Savola K, Lounamaa R, llonen J, et al. (1999) Clinical, autoimmune, and genetic characteristics of very young children with type 1 diabetes. Childhood Diabetes in Finland (DiMe) Study Group. Diabetes Care 22: 1950-1955. 